6-2020

Inferior vena cava injuries - A clinical review

Zia Ur Rehman

Follow this and additional works at: https://ecommons.aku.edu/pakistan_fhs_mc_surg_surg

Part of the Cardiology Commons, Surgery Commons, and the Trauma Commons 


\section{Inferior vena cava injuries - a clinical review}

Zia Ur Rehman

\begin{abstract}
Inferior vena caval (IVC) injuries are uncommon and challenging to treat. Less than $5 \%$ of patients with penetrating abdominal trauma and less than $0.5 \%$ of patients with blunt abdominal trauma have this injury. Patient can present with intraperitoneal haemorrhage or with a contained retroperitoneal haematoma. Mostly it is associated with other abdominal structures injuries. Most commonly injured segment is infrarenal IVC. Operative strategy is different for each segment of injured vein. Infrahepatic injuries are exposed by medial visceral rotation. Retrohepatic and suprahepatic injuries need infrequent exposures. Mortality remains high and range between $31-51 \%$ for the patient brought alive to the operative room. Glasgow coma scale, level of injury, haemodynamic status at presentation and free blood in the peritoneal cavity are some of the predictive factors for mortality in these patients.
\end{abstract}

Keywords: Venous injuries, Penetrating injuries, Abdominal vascular trauma.

DOI: https://doi.org/10.5455/JPMA.21107

\section{Introduction}

Inferior vena caval (IVC) injuries is one the most difficult injuries to deal with. Fortunately IVC is well protected in the abdomen due to its deep anatomical location. Still it is one of the most commonly injured vascular structures during abdominal injuries. ${ }^{1}$

Less than $5 \%$ of the patients with penetrating abdominal injuries have IVC injuries while less than $0.5 \%$ of blunt abdominal trauma patients have these injuries. ${ }^{2}$ It can also be injured iatrogenically. This happen especially while doing dissection for retroperitoneal tumour encasing or adherent to IVC. This can also happen during abdominal aortic aneurysm repair in situations of ruptured aortic aneurysm or in re-do operations when the dissecting planes are not so clear and difficult to find. The incidence has been reported to be1.9\%.3

In the trauma settings, most of the patients die at the ............................................. Section of Vascular Surgery, Department of Surgery, Aga Khan University Hospital, Karachi, Pakistan.

Correspondence: Email: ziaur.rehman@aku.edu scene and are not able to reach the hospital. Out of them who are able to reach, half of them die within next 24 hours due to the sequel of massive bleeding and multi organ failure.

Despite the improved pre-hospital care and transportation and advances in surgical techniques and perioperative care, mortality has not changed over the decades. One possible reason for this can be that patients who previously die at the scene are brought to hospital and on the verge of dying. ${ }^{4}$ This has kept the mortality constant over the decades.

\section{Clinical Anatomy}

IVC is the largest vein in abdomen and runs in the retroperitoneal space to the right of the aorta. It is formed by the confluence of both iliac veins at the level of $L 5$. It moves upwards and receives multiple lumbar veins in the posterior aspect, and a gonadal vein at lateral aspect. The two renal veins join it at the level of L2. It then passes behind the right lobe of liver. Here it receives multiple hepatic veins in and then from the abdomen to the thoracic cavity through diaphragmatic hiatus at the level T8. It drains into right atrium.

IVC is anatomically divided into four segments from injury point and different strategy used for exposure for each of the segment: infra renal, juxta-renal, retrohepatic and supra-hepatic. It is important to identify the site of injury as early as possible in the management of patient because exposure for each segment is different.

\section{Surgical Approaches}

Midline laparotomy is performed in any patient suspected of IVC injury and presenting with acute abdomen or haemodynamic instability. Upon exploration, there can be either active bleeding or retroperitoneal haematoma. The presence of free blood in the peritoneal cavity in not a good prognostic factor. ${ }^{5}$ It is difficult to locate exact site of diffuse venous bleeding compared to pulsatile arterial bleed where spurter can help to locate it. Second, even after the proximal and distal control, the bleeding continues. It may be coming from lumbar veins or side branches and making it difficult for the operator to locate. The first step in case of free blood in the peritoneal cavity is to control by direct compression. Compression of the 
vena cava proximally and distally is an important part of haemorrhage control. 6 This can be helped by spongesticks. Even with adequate compression, haemorrhage can be significant from renal and lumbar venous bleeding. The proximal control can also be achieved by controlling/compressing both iliac veins lateral to L5 vertebra. Manual compression of the proximal aorta below the diaphragm can also provide tamponade and help in locating the site of bleeding. This can be achieved by exposing the supracoelic aorta after dividing gastrohepatic ligament.

Patients presenting with retroperitoneal haematoma will have visible bluish bulge on the right side of the body. It will be pushing intraperitoneal structure anteriorly. For the juxta-hepatic injuries in the supramesocolic region, exposure is achieved by taking the hepatic flexure of colon down. Right colon is dissected to medial side and the medial mobilization of the duodenum is performed. Infra-renal IVC injuries can be exposed by medial rotation of ascending colon. Due to difficult and time consuming exposure of the retrohepatic vena cava, it is better to avoid it if there is no active bleeding or the haematoma is not expanding. They are left to stablilize with effective peri-hepatic packing. If the bleeding is uncontrollable despite packing or the haematoma is expanding, it needs exploration. Exploration needs complete hepatic mobilization and total hepatic vascular isolation. It is time consuming and there is little control. Atriocaval shunt and venovenous bypass can be an alternative in this situation.

\section{Atriocaval Shunt}

In atriocaval, right atrium is controlled after performing sternotomy. The atrial appendage is secured with a purse string suture. It is incised and wide bore chest tube or endotracheal tube with extra side holes is placed beyond the renal veins. It is secured by placing tourniquets, one in the intra pericardial portion of IVC and other in the suprarenal portion of IVC. Vents created in the chest tube below and above the ligated points give adequate flow. It can be a life-saving option. Shunt needs to cross the injured segment. Bruch et al. managed 31 patients with this method.7 Only six patients survived (19\%), all of those patients who survived had gunshot wounds. The results even with use of atriocaval shunt remains dismal. This is due to lack of planning and anticipating the injury

\section{Ligation versus repair}

In a stable patient, vena caval injury can be repaired. It is simple to primarily repair the injury if it involves only the anterior wall. In patients with concomitant anterior and posterior injuries, first the posterior wall is repaired. This can be facilitated by increasing the venotomy and repairing the injury from inside. Then the anterior wall is repaired. 8 Theoretically, interposition grafting may be necessary for larger injuries to avoid stenosis after the repair. ${ }^{9}$ In practice, there are two options: for simple injury, primary repair and for the complex, ligation. Satinsky side biting clamps can also aid in the repair of linear caval injuries and facilitate suture placement in a relatively bloodless field. Lacerations and puncture wounds should be sutured using permanent monofilament suture. Using Teflon felt or skeletal muscle pledgets can aid in haemostasis if the vein wall is friable or if initial suture attempts pull through the vein wall.

As discussed, ligation of IVC is an acceptable solution in case of complex injury as damage control maneuver. ${ }^{10-12}$ It is well tolerated by the patients. Most of the patients do not require fasciotomies. Patients may develop leg swelling postoperatively but it settles with time. In a comprehensive review on IVC injuries ligation was performed in $25 \%$ cases. The ligated patients showed good results without minimal lower extremity oedema. ${ }^{13}$

\section{Endovascular Techniques}

Endovascular techniques and therapies are now being advocated for use in these injuries. In 2014, Briggs $S$ et al. published a case report of retrohepatic injury managed with stent-graft. Endovascular balloon occlusion as a lifesaving technique for occlusion of the IVC with subsequent reduction of massive haemorrhage is now emerging. ${ }^{14}$ The study results of an animal experimental study demonstrated effectiveness of endovenous balloons in retrohepatic IVC injuries in ablating massive haemorrhage. ${ }^{14}$ Its use in clinical setting is awaited.

\section{Factors Associated with Mortality}

The outcomes of these patients presenting with IVC injuries is dependent on the haemodynamic status of the patients at presentation, associated injuries and the anatomical involved segment of IVC. In a haemodynamically unstable patient the goal of treatment is to rapidly control bleeding.

The most important factor associated with mortality is the location of injuries. The mortality increases as the level of injury increases. In one of series mortality rates of $100 \%$ was observed for suprahepatic, 78\% for retrohepatic, and $33 \%$ for suprarenal injuries 9 . The other factor deciding this high mortality rate is number of associated injuries and haemodynamic status of patient at presentation. ${ }^{15,16}$ Maciel JD et al. showed mortality in patients brought alive to hospital with penetrating injuries and operated for IVC injuries was 58\%. ${ }^{17}$ Authors noted that the non-survivors presented with hypotension, and had undergone resuscitative thoracotomy. They did notice that 
retrohepatic injury was more common in non-survivors. ${ }^{17}$ Independent predictor of mortality was the absence of spontaneous tamponade at the time of laparotomy. Glasgow Coma Scale (GCS) is also described as an independent predictor of mortality in IVC trauma. ${ }^{18}$

Von Rooyen et al. in 2015 published a case series. In which they reviewed the outcomes of patients treated over 7 years in a trauma centre in South Africa. All of the IVC injuries was caused by gunshot injuries and most common method of repair was venoraphy. ${ }^{19}$

\section{Conclusion}

IVC injuries are challenging and are associated with high mortality. GCS, level of injury, haemodynamic status and free blood in the peritoneal cavity are some of the predictive of mortality. Knowledge of resuscitation and operative approaches is important for the surgeons involved in care of these patients. Timely intervention and damage control maneuvers can save a lot of these patients.

Disclaimer: Nil.

\section{Conflict of Interest: None.}

Funding Sources: Nil.

\section{References}

1. Asensio JA, Chahwan S, Hanpeter D, Demetriades D, Forno W, Gambaro E, et al. Operative management and outcome of 302 abdominal vascular injuries. Am J Surg. 2000; 180:528-33.

2. Buckman RF, Pathak AS, Badellino MM, Bradley KM, et al. Injuries of the inferior vena cava. Surg Clin North Am. 2001; 81:1431-47.

3. Hans SS, Vang S, Sachwani-Daswani G. latrogenic Major Vascular Injury is associated with increased morbidity of aortic reconstruction. Ann Vasc Surg. 2018; 47:200-4.

4. Eachempati SR, Robb T, Ivatury RR, Hydo LJ, Barie PS. Factors associated with mortality in patients with penetrating abdominal vascular trauma. J Surg Res. 2002; 108:222-6.

5. Kuehne J, Frankhouse J, Modrall G, Golshani S, Aziz I, Demetriades $D$, et al. Determinants of Survival after Inferior Vena Cava Trauma.
Am Surg. 1999; 65:976-81.

6. Hornez E, Béranger F, Monchal T, Baudouin Y, Boddaert G, De Lesquen $\mathrm{H}$, et al. Management specificities for abdominal, pelvic and vascular penetrating trauma. J Visc Surg. 2017; 2: S1878-7886.

7. Burch JM, Feliciano DV, Mattox KL. The Atriocaval Shunt Facts and Fiction.Ann Surg. 1988; 207:555-68.

8. Feliciano DV. Abdominal vascular injuries. Surg Clin North Am 1988:68;741-55

9. Nguyen T, Kalish J, Woodson J. Management of Civilian and Military Vascular Trauma: Lessons Learned. SeminVasc Surg. 2010; 23:235-42.

10. Malgras B, Prunet B, Lesaffre X, Boddaert G, Travers S, Cungi PJ, et al. Damage control: Concept and implementation. J Visc Surg. 2017; 154:S19-S29.

11. Roberts DJ, Bobrovitz N, Zygun DA, Ball CG, Kirkpatrick AW, Faris $P D$, et al. Indications for useof damage control surgery and damage control interventionsin civilian trauma patients: a scoping review. J Trauma Acute Care Surg. 2015; 78:1187-96.

12. Roberts DJ, Bobrovitz N, Zygun DA, Ball CG, Kirkpatrick AW, Faris PD, et al. Indications for useof thoracic, abdominal, pelvic, and vascular damage control interventions in trauma patients: a content analysis andexpert appropriateness rating study. J Trauma Acute Care Surg.2016;79:568-79.

13. Sullivan PS, Dente CJ, Patel S, Carmichael M, Srinivasan JK, Wyrzykowski $A D$, et al. Outcome of ligation of the inferior vena cava in the modern era. Am J Surg. 2010; 199:500-6.

14. Reynolds CL, Celio AC, Bridges LC, Mosquera C, O?Connell B, Bard $M R$, et al. REBOA for the IVC? Resuscitative balloon occlusion of the inferior vena cava (REBOVC) to abate massive hemorrhage in retrohepatic vena cava injuries. J Trauma Acute Care Surg. 2017; 83:1041-6.

15. Rosengart MR, Smith DR, Melton SM, May AK, Rue LW 3rd, et al. Prognostic factors in patients with inferior vena cava injuries. Am Surg. 1999; 65:849-55.

16. Bowley DMG, Degiannis E, Goosen J, Boffard KD. Penetrating vascular trauma in Johannesburg, South Africa. Surg Clin N Am. 2002; 82:221-36.

17. Maciel JD, Plurad D, Gifford E, deVirgilio C, Koopmann M, Neville A, et al. Predictors of Mortality in Patients with Penetrating Inferior Vena Cava Injuries Surviving to the Operating Room.Am Surg. 2015;81:1000-4.

18. Cudworth M, Fulle A, Ramos JP, Arriagada I. GCS as a predictor of mortality in patients with traumatic inferior vena cava injuries: a retrospective review of 16 cases.World J Emerg Surg. 2013; 8:59.

19. van Rooyen PL, Karusseit VO, Mokoena T. Inferior vena cava injuries: a case series and review of the South African experience. Injury. 2015; 46:71-5. 